\title{
Mycoplasma-related endobacteria within Mortierellomycotina fungi: diversity, distribution and functional insights into their lifestyle
}

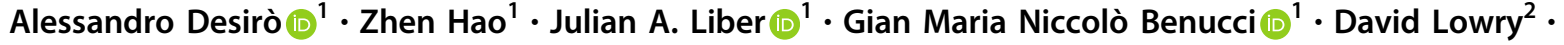 \\ Robert Roberson ${ }^{2} \cdot$ Gregory Bonito iD ${ }^{1}$
}

Received: 27 August 2017 / Revised: 22 December 2017 / Accepted: 10 January 2018 / Published online: 23 February 2018

(c) International Society for Microbial Ecology 2018

\begin{abstract}
Bacterial interactions with animals and plants have been examined for over a century; by contrast, the study of bacterial-fungal interactions has received less attention. Bacteria interact with fungi in diverse ways, and endobacteria that reside inside fungal cells represent the most intimate interaction. The most significant bacterial endosymbionts that have been studied are associated with Mucoromycota and include two main groups: Burkholderia-related and Mycoplasmarelated endobacteria (MRE). Examples of Burkholderia-related endobacteria have been reported in the three Mucoromycota subphyla. By contrast, MRE have only been identified in Glomeromycotina and Mucoromycotina. This study aims to understand whether MRE dwell in Mortierellomycotina and, if so, to determine their impact on the fungal host. We carried out a large-scale screening of 394 Mortierellomycotina strains and employed a combination of microscopy, molecular phylogeny, next-generation sequencing and qPCR. We detected MRE in 12 strains. These endosymbionts represent novel bacterial phylotypes and show evidence of recombination. Their presence in Mortierellomycotina demonstrates that MRE occur within fungi across Mucoromycota and they may have lived in their common ancestor. We cured the fungus of its endosymbionts with antibiotics and observed improved biomass production in isogenic lines lacking MRE, demonstrating that these endobacteria impose some fitness costs to their fungal host. Here we provided the first functional insights into the lifestyle of MRE. Our findings indicate that MRE may be antagonistic to their fungal hosts, and adapted to a non-lethal parasitic lifestyle in the mycelium of Mucoromycota. However, context-dependent adaptive benefits to their host at minimal cost cannot not be excluded. Finally, we conclude that Mortierellomycotina represent attractive model organisms for exploring interactions between MRE and fungi.
\end{abstract}

\section{Introduction}

Mortierellomycotina are a group of early-diverging fungi that belong to the phylum Mucoromycota [1]. The subphylum Mortierellomycotina is constituted by one order,

Electronic supplementary material The online version of this article (https://doi.org/10.1038/s41396-018-0053-9) contains supplementary material, which is available to authorized users.

$\triangle$ Alessandro Desirò

adesiro@msu.edu

bonito@msu.edu

1 Department of Plant Soil and Microbial Sciences, Michigan State University, East Lansing, MI, USA

2 School of Life Sciences, Arizona State University, Tempe, AZ, USA one family and eight or nine genera that are not completely resolved [2]. Taxa within Mortierellomycotina show a significant diversity with a still undefined number of over 100 species [3] that are distributed among seven major clades/groups [4]. Species in this group, especially lineages in the genus Mortierella, have a global distribution, occur in a wide range of habitats, and are often dominant in soil fungal communities [5]. These fungi are common soil dwellers, frequently associated with plant rhizospheres [68], from which many species can be easily isolated.

Fungi in Mucoromycota display several distinctive morphological and ecological traits [1]. For example, they possess a coenocytic mycelium, with only occasional septa. In addition, diverse lineages of this phylum can harbor endobacteria. The presence of bacteria within the fungal mycelium forces us to reconsider concepts of fungal microbiomes [9] and holobionts, that is, the fungal host plus its microbial symbionts [10]. In particular, two main groups 
of endobacteria are known to be associated with Mucoromycota: Betaproteobacteria (Burkholderia-related) and Mollicutes (Mycoplasma-related) [11].

Examples of Burkholderia-related endobacteria (BRE) have been reported in each of the three Mucoromycota subphyla. Fungi in Gigasporaceae (Glomeromycotina) can host Candidatus Glomeribacter gigasporarum [12] whose presence appears to enhance fungal host fitness [13]. Mortierella elongata (Mortierellomycotina) can associate with Mycoavidus cysteinexigens [14], which strongly impacts the metabolism and reduces the growth rate of its host $[15,16]$. Rhizopus microsporus (Mucoromycotina), the causal agent of rice seedling blight, can harbor Burkholderia rhizoxinica [17], which produces potent phytotoxins used by the fungus to attack and cause disease in rice seedlings [18].

The second group of endosymbionts that can be found in Mucoromycota are Mycoplasma-related endobacteria (MRE). Even though their presence within Glomeromycotina has been known for a long time [19, 20], MRE have only recently been identified as a novel group of Mollicutes, phylogenetically related to Entomoplasmatales and Mycoplasmatales [21]. MRE are coccoid bacteria that cannot grow outside of their fungal host, thus they are obligate endosymbionts. They were first described in Glomeromycotina, where they can be found inside spores of almost all major lineages [9, 21, 22], but also within hyphae colonizing hornworts [23], lycopods [24] and liverworts [25]. Recently, MRE from Glomeromycotina were accommodated within the novel species Candidatus Moeniiplasma glomeromycotorum ( $\mathrm{CaMg}$ ) [26]. The genomes of $C a \mathrm{Mg}$ have been sequenced and show a highly reduced gene content, indicative of metabolic dependence on its host, and therefore consistent with the yet-to-be-cultured status of these microbes [27, 28]. $\mathrm{Ca} \mathrm{Mg}$ possesses a remarkably plastic genome, a feature related to the maintenance of recombination machinery and mobile genetic elements [27, 29]. These two factors, together with an ultrarapid mutation rate, horizontal gene transfer and horizontal transmission contribute to the extraordinary diversity that characterizes these bacteria [9, $21,22,27,29,30]$. The function of this group of endobacteria have never been assessed, but it has been suggested that $\mathrm{CaMg}$ may be parasites of Glomeromycotina [22].

The presence of Mollicutes endosymbionts is not limited to Glomeromycotina. MRE were also identified inside sporocarps of several specimens of Endogone and Jimgerdemannia [31] (Endogonaceae, Mucoromycotina) [32]. These findings pushed Desirò and colleagues [32] to hypothesize that the interaction with MRE originated before the diversification of their fungal hosts (Glomeromycotina and Mucoromycotina). Codivergence patterns between MRE and the two host fungal lineages implies that the fungal association with MRE may indeed predate the divergence of the eukaryotic hosts [22]. Such a scenario is in line with an early MRE invasion hypothesis that assumes the existence of an ancestral MRE in the common ancestor of Glomeromycotina and Mucoromycotina [11]. However, divergence time estimates indicate that the diversification of Glomeromycotina and Mucoromycotina [16, 33] predates the origin of Entomoplasmatales and Mycoplasmatales (thus MRE) [22, 34], in support of the alternative late MRE invasion hypothesis [11].

Irrespective on when MRE established in fungi, these endosymbionts appear to have found a suitable habitat within Mucoromycota. However, such a claim needs further confirmation since MRE have not been detected in Mortierellomycotina thus far. Mortierellomycotina share several morphological and ecological similarities with the other two Mucoromycota subphyla, some of whose members are known to harbor MRE. Moreover, BRE have recently been detected within Mortierellomycotina [14, 16, 35]. For these reasons, we hypothesized that Mortierellomycotina may also host MRE. To test this hypothesis, we carried out a large-scale study (394 strains) of Mortierellomycotina fungi employing a combination of microscopy, molecular phylogeny, next-generation sequencing, qPCR and fungal biomass production assays.

Here we described for the first time the presence of MRE in fungi of the subphylum Mortierellomycotina. Novel MRE phylotypes were identified in the mycelium of several phylogenetically diverse strains of Lobosporangium and Mortierella, demonstrating that MRE are present within the three Mucoromycota subphyla (Glomeromycotina, Mortierellomycotina and Mucoromycotina). Furthermore, we obtained isogenic lines of fungi cured of their endobacteria and provided the first functional evidence on the role of MRE as potential antagonists of fungi adapted to a non-lethal parasitic lifestyle in the mycelium of Mucoromycota.

\section{Materials and methods}

Complete details of each experimental procedure are available in the Supplementary Text S1.

\section{Fungal isolation}

Modified versions of the soil-plate [36] and selectivebaiting methods [37] were used to isolate Mortierellomycotina from soils. Mortierellomycotina were also isolated from pine and spruce roots using a previously described method [38]. Other fungal strains were obtained from collaborators and public collections (Supplementary Table S1). 
Table 1 List of Mortierellomycotina strains that host MRE

\begin{tabular}{|c|c|c|c|c|c|c|}
\hline Species & Strain & Origin & Phylogroup & MRE OTU & Cured line & Mycoavidus \\
\hline Lobosporangium transversale & NRRL 5525 & Texas, USA & Group 5 & OTU 3 & - & - \\
\hline Mortierella capitata & AD192 & Kenya & Group 5 & OTU 4 & $\checkmark$ & - \\
\hline Mortierella elongata & AD073 & Michigan, USA & Group 7 & OTU 1 & $\checkmark$ & $\checkmark$ \\
\hline Mortierella elongata & NVP80 & Michigan, USA & Group 7 & OTU 1 & $\checkmark$ & - \\
\hline Mortierella gamsii & AM1032 & Illinois, USA & Group 7 & OTU 1 & - & - \\
\hline Mortierella humilis & AD092 & Pennsylvania, USA & Group 2 & OTU 1 & $\checkmark$ & - \\
\hline Mortierella minutissima & KOD988 & Illinois, USA & Group 2 & OTU 1 & - & $\checkmark$ \\
\hline Mortierella rostafinskii & NRRL A-17819 & - & Group 5 & OTU 1 & - & - \\
\hline Mortierella selenospora & CBS 811.68 & Netherlands & Group 1 & OTU 3 & $\checkmark$ & - \\
\hline Mortierella thaxteri & NRRL 25716 & Georgia, USA & Group 6 & OTU 1 & - & - \\
\hline Mortierellomycotina sp. & AD185 & Kenya & Group 8 & OTU 2 & $\checkmark$ & - \\
\hline Mortierellomycotina sp. & GBAus27b & Australia & Group 2 & OTU 2 & $\checkmark$ & - \\
\hline
\end{tabular}

Species, strain name/number, place of origin, phylogenetic grouping (sensu [4]), operational taxonomic unit of MRE based on a 94\% sequence similarity criterion, availability of an isogenic line cured of MRE, and presence of Mycoavidus are shown for each strain

\section{DNA extraction, amplification, and clone library analysis}

Fungal genomic DNA was extracted using a CTAB-based method [39]. The fungal ITS region and partial 28S rRNA gene were amplified using primers ITS1F [40] and LR3 [41]. Partial MRE 16S rRNA gene was amplified using primers 109F [21] and 1387Rmod [42] or 1492R [43]. MRE amplicons were cloned using TOPO-TA cloning kit (Thermo Fisher Scientific).

\section{Sequence analysis and phylogenetic reconstructions}

Sequences were assembled and edited in Geneious v.8.1.7 [44], and aligned with MAFFT [45] or MUSCLE [46]. Fungal and MRE alignments were trimmed with GBlocks v.0.91b [47]. Best-fit nucleotide substitution models were estimated with jModelTest v.2.1.9 [48]. Phylogenetic reconstructions were carried out with MrBayes v.3.2.6 [49] and RAxML v.8.2.4 [50]. Identification of recombination breakpoints was conducted with the Genetic Algorithm for Recombination Detection [51].

\section{Illumina library preparation, MiSeq sequencing and data analysis}

The V4 region of the 16S rRNA gene was amplified using primers 515F and 806R [52]. Amplification, adapter ligation and amplicon barcoding were carried out using a modified version of a previously described method [53].

\section{Clearing of MRE from Mortierellomycotina}

MRE were removed from wild-type (WT) strains of Mortierellomycotina using a combination of four antibiotics: 80 $\mu \mathrm{g} / \mathrm{ml}$ Ampicillin, $50 \mu \mathrm{g} / \mathrm{ml}$ Kanamycin, $50 \mu \mathrm{g} / \mathrm{ml}$ Streptomycin, and $120 \mu \mathrm{g} / \mathrm{ml}$ Ciprofloxacin.

\section{Growth conditions and sample preparation}

To test the effect of temperature on fungal biomass production and MRE abundance, cured and WT lines of $M$. elongata NVP80 were incubated at 4,22 and $37^{\circ} \mathrm{C}$. To test the effect of the media type on fungal biomass production and MRE abundance, the two lines were grown in six nutrient-rich and seven nutrient-poor liquid media types (Supplementary Table S2). Similarly, cured and WT lines of M. selenospora CBS 811.68 and Mortierellomycotina sp. GBAus $27 \mathrm{~b}$ were incubated at $22^{\circ} \mathrm{C}$. The mycelia were then collected, freeze-dried, and dry weights were measured.

\section{Quantification of MRE cells}

Mortierella elongata NVP80 was selected in order to quantify the amount of MRE cells in the fungal mycelium grown at different temperatures and in different media types. The new primers MortMREqAD1f and MortMREqAD1 $r$ were used to target the MRE 16S rRNA gene. Template PCR amplicons containing the target DNA sequence were used to generated a standard curve as an external standard as previously described $[9,54]$. 


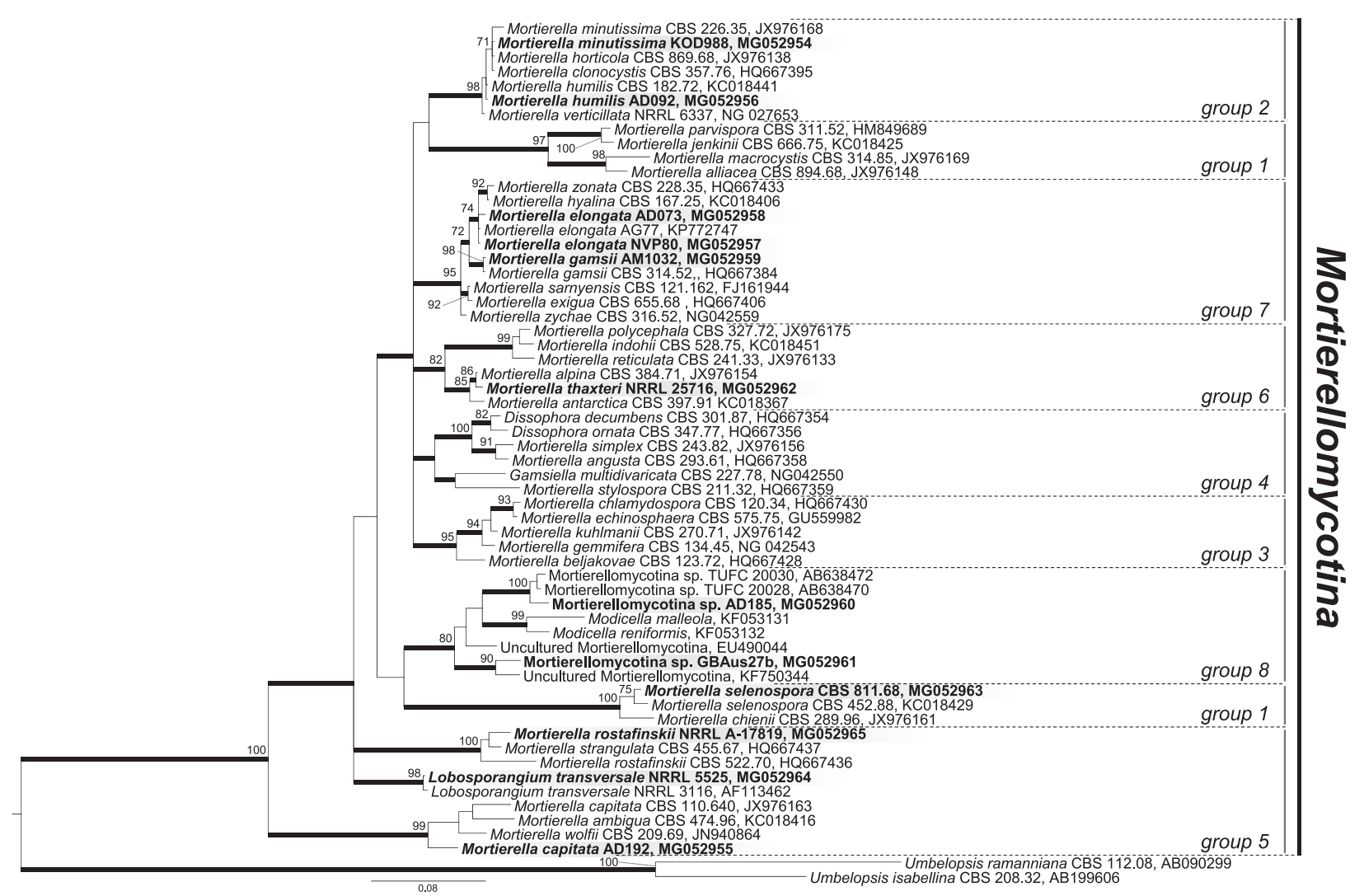

Fig. 1 Phylogenetic placement of the 12 strains of Mortierellomycotina found to harbor MRE within the eight Mortierellomycotina phylogenetic groups (sensu [4]) based on 28S rRNA gene sequences. The 12 strains are spread across five Mortierellomycotina phylogenetic groups (groups 1, 2, 5, 6, 7) (sensu [4]) and

\section{Statistics}

Statistical analyses and graphs were performed using the computing environment $\mathrm{R}$ [55].

\section{Ultrastructural analyses}

Mortierella elongata NVP80 and M. humilis AD092 were subjected to high-pressure freeze-substitution in order to preserve fungal and bacterial ultrastructure, and observed under a transmission electron microscope.

\section{Results}

\section{Identity and diversity of MRE and their Mortierellomycotina hosts}

This study is the first to assess the diversity and distribution of MRE in Mortierellomycotina. In total, we investigated 394 fungal strains originated worldwide and including lineages belonging to each of the seven phylogenetic groups within the additional phylogenetic group 8/Modicella group. The tree shows the topology obtained with the Bayesian method; branches with Bayesian posterior probabilities $\geq 0.95$ are thickened and ML bootstrap support values $\geq 70$ are shown. Sequences generated in this study are in bold and highlighted in gray

of Mortierellomycotina delimited by Wagner and colleagues [4] (Supplementary Table S1). Among them, only 12 strains were found to harbor MRE (Table 1). Phylogenetic reconstructions placed 10 of these strains within the genera Lobosporangium and Mortierella. In detail, several species were identified: $L$. transversale, $M$. capitata, $M$. elongata, M. gamsii, M. humilis, M. minutissima, M. rostafinskii, M. selenospora and $M$. thaxteri. These species were spread across five of the seven Mortierellomycotina phylogenetic groups (sensu [4]). Interestingly, the remaining two strains (AD185 and GBAus27b) clustered within an additional phylogenetic group that includes Modicella spp. Smith et al. [56] and undescribed Mortierellomycotina taxa, which we refer to as group 8/Modicella group (Fig. 1). Fungal rDNA sequences generated in this study are available on GenBank (MG052954-MG052965).

A MRE-specific primer pair was used to screen for MRE, preventing the detection of other endobacteria lineages that can reside within Mortierellomycotina (i.e. Mycoavidus cysteinexigens). The stringent chimera screen did not detect any PCR-generated artifacts. In contrast to Endogonaceae and Glomeromycotina, neither cloned 16S rDNA sequences 


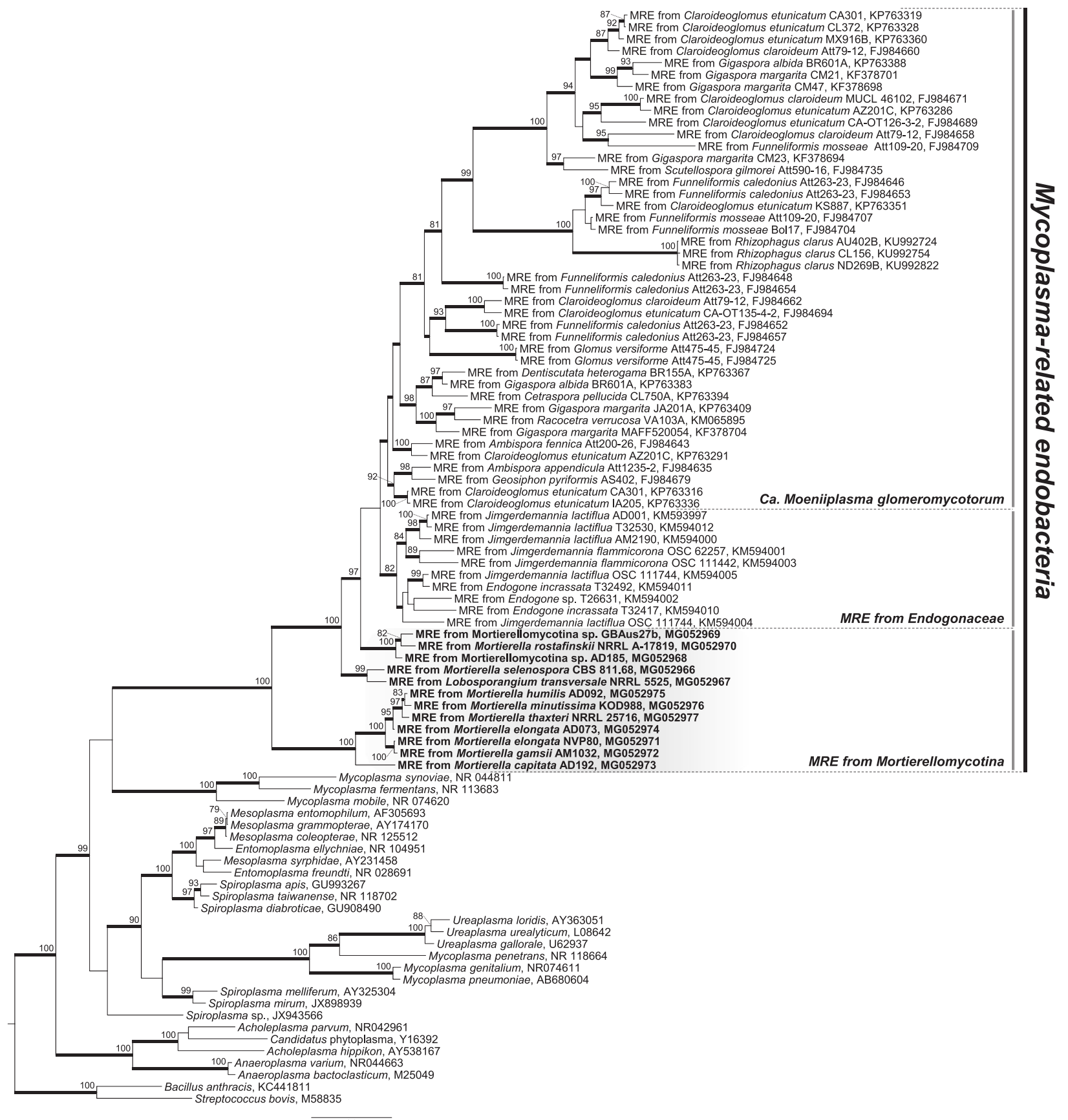

Fig. 2 Phylogenetic placement of MRE identified in Mortierellomycotina based on 16S rRNA gene sequences. MRE from Mortierellomycotina cluster into three novel early-diverging clades within the monophyletic clade that encompasses $\mathrm{Ca}$. Moeniiplasma glomeromycotorum and MRE from Endogonaceae. A single MRE 16S rDNA phylotype was obtained from each fungal strain. The tree shows the topology obtained with the Bayesian method; branches with Bayesian posterior probabilities $\geq 0.95$ are thickened and ML bootstrap support values $\geq 70$ are shown. Sequences generated in this study are in bold and highlighted in gray

nor Illumina MiSeq reads (Illumina MiSeq reads were not generated for M. capitata AD192) retrieved from Mortierellomycotina showed evidence of intrahost MRE sequence divergence. That is, only a single MRE $16 \mathrm{~S}$ rDNA phylotype was obtained from each fungal strain (Fig. 2; Supplementary Figure S1). The clustering of all cloned MRE sequences from Mortierellomycotina using a $94 \%$ sequence similarity criterion (typically used for Mollicutes species delimitation) resulted in four operational taxonomic units (OTUs) (Table 1; Supplementary Figure S1). Together, MRE from different strains exhibited up to $19 \%$ interhost sequence divergence. Furthermore, they showed sequence similarity values $<94 \%$ when compared to MRE sequences obtained from Endogonaceae and Glomeromycotina: the 
Fig. 3 Mortierella elongata NVP80 cured a, $\mathbf{c}$ and wildtype $\mathbf{b}, \mathbf{d}$ lines grown in ME + YE broth and agar plates $\mathbf{a}, \mathbf{b}$ and PD broth and PD+YE agar plates $\mathbf{c}, \mathbf{d}$. The cured line shows greater biomass production and more aerial hyphae than the wild-type line
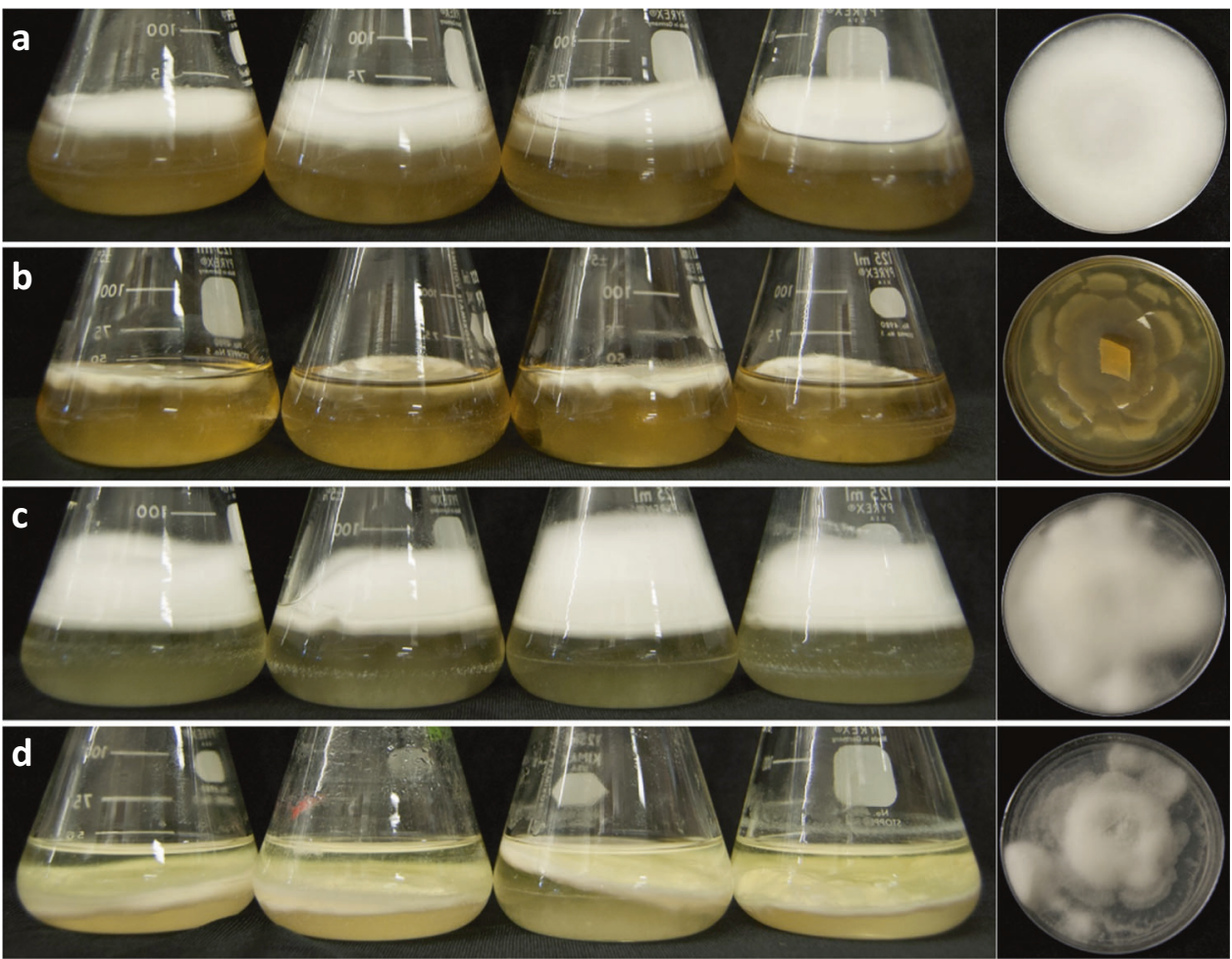

least similar MRE sequences were retrieved from several strains of Rhizophagus clarus ( $74 \%$ sequence similarity with MRE sequence included in OTU1), whereas the most similar were generated from Glomeromycotina lineages such as Ambispora appendicula, Ambispora fennica and Geosiphon pyriformis, and Jimgerdemannia lactiflua ( $92 \%$ sequence similarity with MRE sequences included in OTU2 and OTU3). Regardless of the high level of sequence divergence, phylogenetic reconstructions placed MRE sequences from Mortierellomycotina into three novel earlydiverging clades within a well-supported monophyletic clade that encompasses MRE from Endogonaceae and Glomeromycotina (Fig. 2). Illumina MiSeq amplicon sequencing results showed that two strains $(M$. elongata AD073 and M. minutissima KOD988) hosted both MRE and Mycoavidus endobacteria. Thus, in this respect, Mortierellomycotina are similar to Glomeromycotina, in that they can harbor multiple types of endobacteria within a single individual [9]. MRE rDNA sequences generated in this study are available on GenBank (MG052966MG052989).

We searched for evidence of recombination in the 1086 nucleotide alignment constituted by the $16 \mathrm{~S}$ rRNA gene sequences of MRE associated with Mortierellomycotina. The Genetic Algorithm for Recombination Detection (GARD) identified two possible recombination breakpoints at positions 118 and 261. The corrected Akaike information criterion (AICc) score for the best-fitting GARD model
(6794), allowing for different topologies of the three segments of the alignment determined by recombination breakpoints was lower than the AICc score for a model that assumed the same topology for all the segments (6824.8) (Supplementary Figure S2). The Kishino-Hasegawa (KH) test confirmed the two recombination breakpoints as resulting in significant topological incongruences between the three alignment segments $(P<0.01)$.

\section{Mortierellomycotina fungi cured of their MRE symbionts}

Using a combination of four antibiotics we cleared MRE from wild-type (WT) Mortierellomycotina fungi obtaining cured isogenic lines. MRE were removed from the following strains: M. capitata AD192, M. elongata AD073 and NVP80, M. humilis AD092, M. selenospora CBS 811.68, and Mortierellomycotina sp. AD185 and GBAus27b (Table 1; Supplementary Figure S3). All cured lines grew faster and generally produced more aerial hyphae when compared to WT lines (Fig. 3).

\section{Effect of temperature on fungal biomass production and MRE abundance}

To evaluate the effect of temperature on fungal biomass production and MRE abundance, we studied cured and WT isogenic lines of $M$. elongata NVP80 grown at 4,22 and $37^{\circ} \mathrm{C}$. 


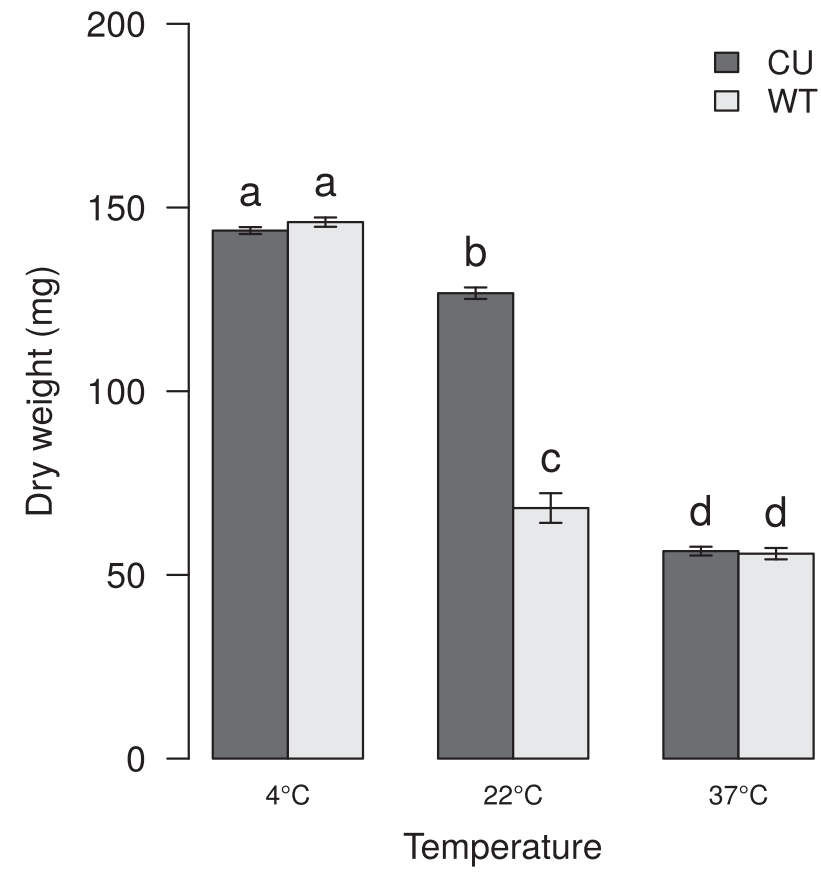

Fig. 4 Mean dry weight (mg) values of M. elongata NVP80 cured (CU) and wild-type (WT) lines grown at different temperature conditions. Bars represent the mean (4 replicates) \pm standard deviation. Lowercase letters represent Tukey honestly significant differences after ANOVA at $P \leq 0.05$

Both lines generated the greatest amount of biomass when incubated at $4{ }^{\circ} \mathrm{C}$, whereas less fungal biomass production occurred at $22^{\circ} \mathrm{C}$ and very little biomass was produced at $37^{\circ} \mathrm{C}$. There were no significant differences in fungal biomass production between cured and WT lines when grown at 4 and $37{ }^{\circ} \mathrm{C}$. Conversely, when maintained at $22{ }^{\circ} \mathrm{C}$, the cured line showed a statistically significantly greater biomass production than the WT line (Fig. 4). Similar results were also observed for $M$. selenospora CBS 811.68 and Mortierellomycotina sp. GBAus27b grown at $22{ }^{\circ} \mathrm{C}$ : cured lines had a considerably greater biomass than WT lines (Supplementary Figure S4).

We quantified with RT-qPCR the relative and absolute number of MRE cells hosted within the mycelium of the WT line of M. elongata NVP80. Quantifications were made for mycelia grown at the three different temperature conditions listed above. The amount of MRE cells within the fungal host was variable between, but also within the three tested temperatures. The highest density (MRE cells per mg of dry mycelium) and absolute number of MRE cells was detected in the mycelia grown at $22{ }^{\circ} \mathrm{C}$ (mean value of $\sim 936,512 \mathrm{MRE}$ cells $/ \mathrm{mg}$, corresponding to a total of $\sim 63$ million MRE cells). Conversely, MRE populations plummeted when the fungus was grown either at 4 or $37^{\circ} \mathrm{C}$, with the lowest amount detected at $37{ }^{\circ} \mathrm{C}$ (mean value of $\sim 3757$ MRE cells/mg, corresponding to a total of $\sim 213,496 \mathrm{MRE}$ cells) (Fig. 5; Supplementary Figure S5).
Effect of media type on fungal biomass production and MRE abundance

To evaluate the effect of media type on fungal biomass production and MRE abundance, we studied cured and WT isogenic lines of $M$. elongata NVP80 grown in 13 different liquid media types at $22^{\circ} \mathrm{C}$. When maintained in nutrientrich media (Fig. 6a), both lines had a significantly higher biomass production in PD than ME-based media, with the highest biomass value measured when grown in PD+YE. When grown in nutrient-poor media (Fig. 6b), maximum biomass production for both lines occurred in 1/10 PD+YE $+\mathrm{PE}$. The cured line produced the greatest amount of biomass on all tested media types. Differences between the two lines were more accentuated when they were grown in media supplemented only with YE. In contrast, when grown in 1/10 ME and 1/10 PD, and/or peptone-supplemented media, the "deficit" of the WT line biomass production was reduced (Fig. 6).

We quantified with RT-qPCR the relative and absolute amount of MRE cells within the mycelium of the WT line of M. elongata NVP80 maintained in seven different media types. As with temperature, the density and absolute amount of MRE cells within the fungal host was variable between, but also within, the tested media types. The highest endobacterial density was detected in 1/10 ME + YE (mean value density of $\sim 3.1$ million MRE cells per $\mathrm{mg}$ ) and was statistically significantly different from the other media types. The lowest average density of MRE was detected in mycelia grown in YE + PE (mean value density of 407,721 MRE cells per mg), although the lowest value for a single replicate was observed in 1/10 ME ( 45,472 MRE cells/mg) (Fig. 7a). The absolute amount of MRE cells was significantly higher in mycelia grown in nutrient-rich compared to nutrient-poor media types. The highest number of MRE cells was detected in ME + YE + PE (mean value of $~ 90$ million MRE cells), whereas the lowest number of cells was observed when mycelia were grown 1/10 ME (mean value of $\sim 12.8$ million MRE cells) (Fig. 7b).

\section{Localization of MRE in the mycelium of Mortierellomycotina}

To complement the molecular detection of MRE in Mortierellomycotina and confirm their cytoplasmic localization within the fungal mycelium, we processed WT lines of $M$. elongata NVP80 and M. humilis AD092 for transmission electron microscopy. We used high pressure and freezesubstitution to preserve fungal and endobacteria ultrastructure. MRE visualized in Mortierellomycotina were coccoid, $310-540 \mathrm{~nm}$ in size, and resided directly within the fungal cytoplasm. Similar to $\mathrm{CaMg}$, a thin homogeneous 


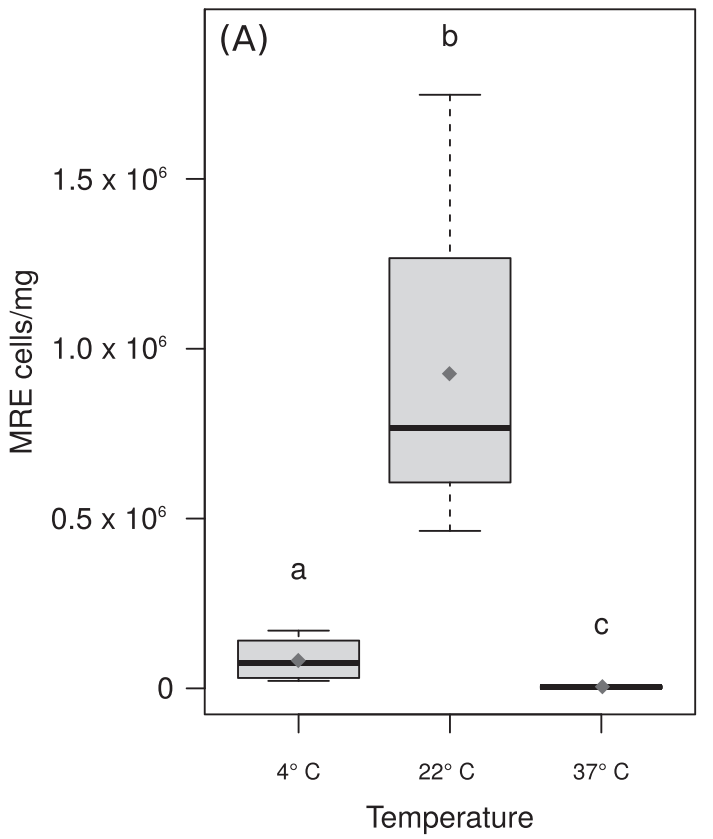

Fig. 5 Distribution of the relative (cells per $\mathrm{mg}$ ) $\mathbf{a}$ and absolute b number of MRE cells within the mycelium of M. elongata NVP80 grown at different temperatures. Boxplots summarize minimum, first quartile, median, third quartile, and maximum values of the data

electron-dense layer was observed outside the bacterial cell membrane (Fig. 8; Supplementary Figures S6). However, the lack of cell wall in Mollicutes [57] and the absence of genes involved in the peptidoglycan synthesis in available draft genomes of $\mathrm{CaMg}[27,28]$ indicate that this structure may be the result of the sample preparation, rather than a Gram-positive cell wall [26]. MRE were mostly present in the older part of the culture, where bacterial cells undergoing cell division were frequently observed (Fig. 8).

\section{Discussion}

This research is the first to describe the presence of MRE in fungi belonging to the subphylum Mortierellomycotina. These endosymbionts represent novel bacterial phylotypes and were detected in the mycelium of Lobosporangium transversale, nine species of Mortierella and two undescribed Mortierellomycotina taxa. Given that Glomeromycotina and Mucoromycotina are known to harbor MRE, our findings demonstrate that MRE are present within all three Mucoromycota subphyla. Our data also indicate that genetic recombination is evident in MRE from Mortierellomycotina. Moreover, having generated isogenic lines of fungi that are cured of their bacterial endosymbionts, we provide the first functional insights into the lifestyle of this cryptic group of endobacteria in their fungal hosts.



distribution. Mean values (red diamond) are also shown. Lowercase letters represent Mann-Whitney significant differences after Kruskal-Wallis test at $P \leq 0.05$

\section{Ca. Moeniiplasma glomeromycotorum and MRE: a single or multiple species?}

Since the first molecular characterization of MRE in Glomeromycotina [21], high level of MRE intra- and interhost diversity has been an intriguing feature of these bacteria. This unexpected diversity appears to be determined by two main factors: an ultrarapid mutation rate that may be attributed to the loss of DNA repair mechanisms [30], and horizontal gene transmission across different MRE genotypes [22, 29]. These peculiar biological features, together with their habitat (i.e., the cytoplasm of Glomeromycotina), are distinctive of $\mathrm{Ca}$. Moeniiplasma glomeromycotorum [26]. Irrespective of the intraspecific 16S rRNA gene sequence similarity level exhibits by this novel bacterial lineage (79\% similarity), which is lower than the $94 \%$ sequence similarity level suggested for delimiting Mollicutes species, $\mathrm{CaMg}$ includes all MRE identified to date in Glomeromycotina. The species definition suggested for $\mathrm{CaMg}$ is in line with previous species delineation proposed for other heritable endobacteria such as Wolbachia pipientis [58]. Indeed, several so-called 'supergroups' of endobacteria from various arthropods and filarial nematodes with a close affinity to $W$. pipientis are formally included in this species. Following the same rationale, very diverse MRE from different Glomeromycotina taxa were grouped into the single species $\mathrm{CaMg}$. But what about MRE harbored in Mortierellomycotina? Should they be included in the species 

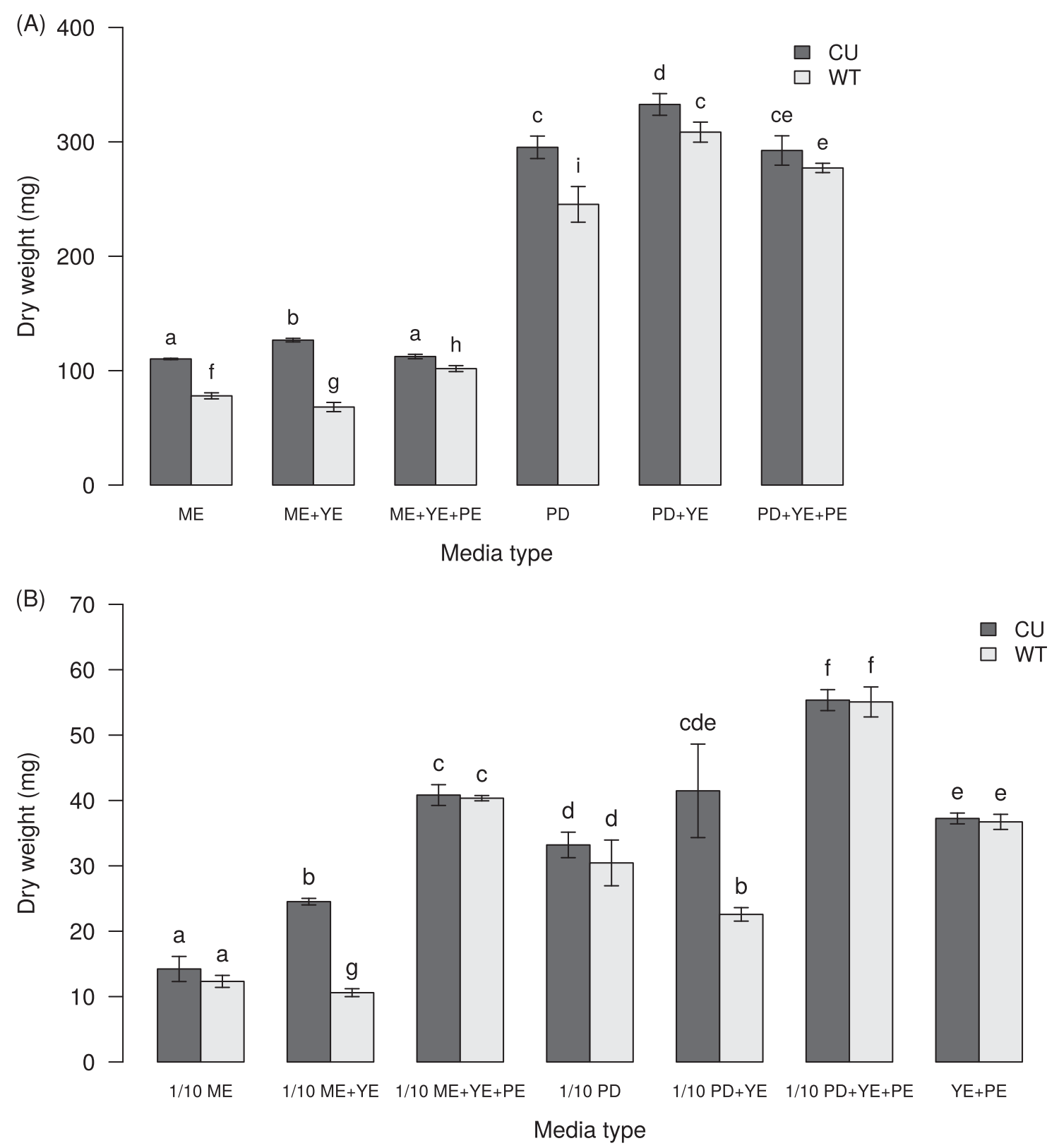

Fig. 6 Mean dry weight $(\mathrm{mg})$ values of $M$. elongata NVP80 cured (CU) and wild-type (WT) lines grown in different rich $\mathbf{a}$ and poor $\mathbf{b}$ media types. Bars represent the mean (4 replicates) \pm standard deviation. Lowercase letters represent Mann-Whitney significant differences after Kruskal-Wallis test at $P \leq 0.05$

most remarkable features displayed by $\mathrm{CaMg}$, appears not to be shared by MRE from Mortierellomycotina. Indeed, each Mortierellomycotina strain examined in our study harbors a unique and homogenous MRE population. Also, including MRE from Mortierellomycotina within the species $\mathrm{CaMg}$ would further drop the already remarkably low level of intraspecific sequence similarity to $\sim 74 \%$. For those reasons, we suggest that MRE from Mortierellomycotina represent a novel $\mathrm{Ca}$. Moeniiplasma taxon related to $\mathrm{CaMg}$. 


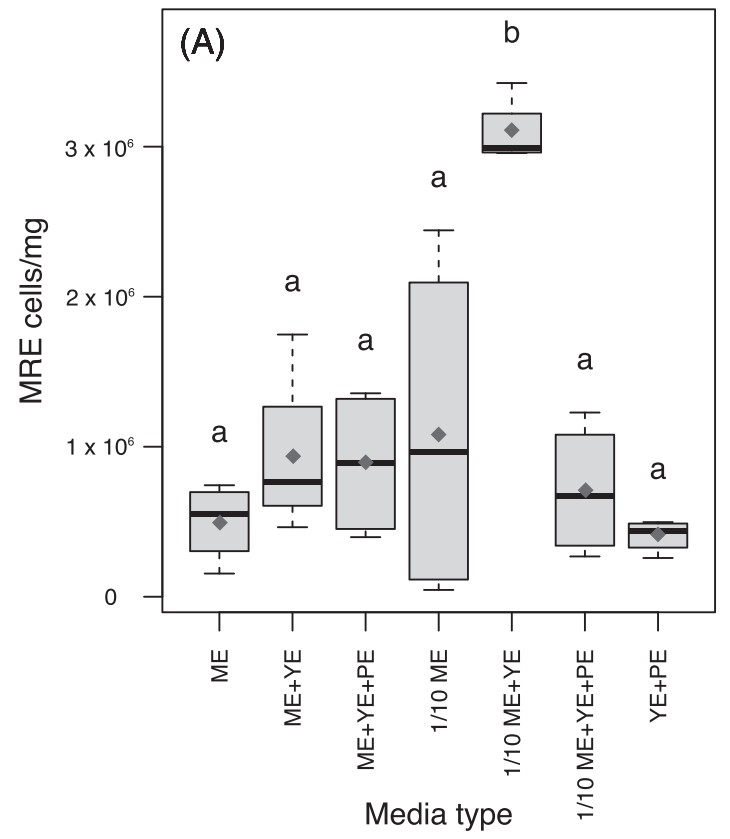

Fig. 7 Distribution of the relative (cells per $\mathrm{mg}$ ) $\mathbf{a}$ and absolute $\mathbf{b}$ number of MRE cells within the mycelium of M. elongata NVP80 grown in different media types. Boxplots summarize minimum, first quartile, median, third quartile, and maximum values of the data

\section{What is the role of endobacteria? Adding the first tile to the tangled MRE puzzle}

Perhaps the most fascinating study describing a function of a bacterial endosymbiont is represented by Burkholderia rhizoxinica, which is responsible of the pathogenicity and its fungal host Rhizopus microsporus [59]. In recent years, several other studies provided novel insights into the lifestyle of fungal endobacteria, mainly Burkholderia-related endobacteria (BRE) [13, 15-17, 60-64]. Importantly, all these contributions became possible after endobacteria were cleared from their hosts [16, 17, 65], thus allowing researchers to compare the same fungal strain in presence/ absence of its BRE symbiont.

In contrast to those on BRE, there are no studies reporting Endogonaceae or Glomeromycotina successfully cured from their MRE endosymbionts. Consequently, it has been impossible to provide experimental evidence on the lifestyle and functions of MRE. Mortierellomycotina are saprotrophic fungi that differ from obligate biotrophs in Glomeromycotina and the still little-known and hard-tohandle Endogonaceae, in which they can easily be grown, propagated and manipulated in pure culture. Our finding of MRE inside the mycelium of Mortierellomycotina make it possible to obtain isogenic fungal lines cured of their MRE, and therefore to provide the first functional insights into the lifestyle of this enigmatic group of bacteria. Indeed, comparing cured and WT lines, we demonstrated that MRE have a negative effect on the biomass production of the

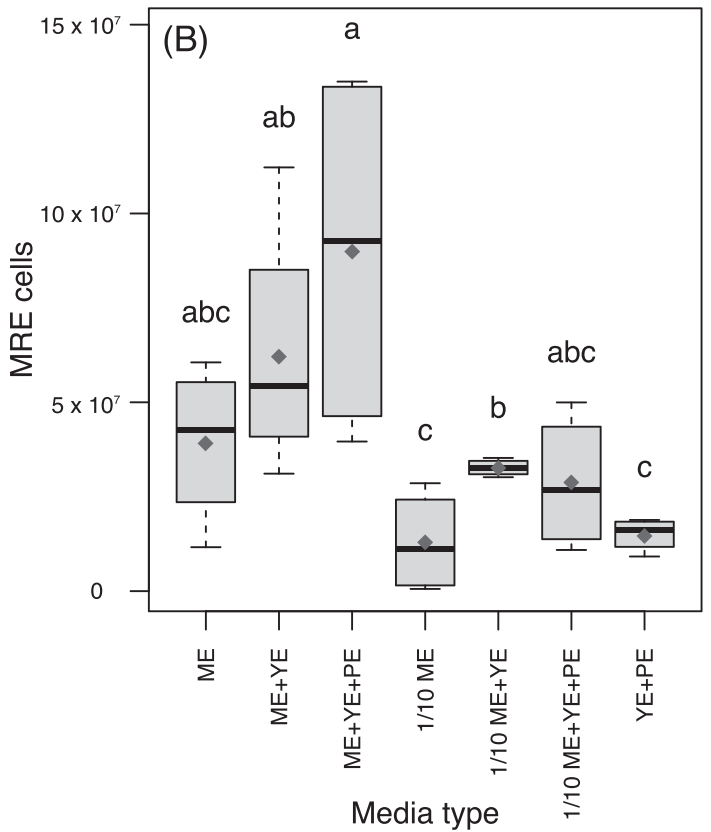

distribution. Mean values (red diamond) are also shown. Lowercase letters represent Mann-Whitney significant differences after Kruskal-Wallis test at $P \leq 0.05$

fungal host, which is dependent on temperature and media type. Furthermore, Illumina MiSeq sequencing results showed that MRE are the only dwellers in the three tested Mortierellomycotina strains and therefore are responsible for the negative changes occurring to their eukaryotic hosts.

Experimental results testing the effect of temperature on fungal biomass production and MRE abundance were particularly intriguing. Indeed, first, we demonstrated that the amount of MRE is temperature dependent. Second, by coupling fungal biomass measurements with MRE quantifications, we showed that the higher the number of MRE cells inside the fungal mycelium, the greater the reduction in biomass of the WT line compared to the cured line. This trend was clearly evident at $22^{\circ} \mathrm{C}$, the condition where we found the highest amount of MRE and the greatest difference in biomass between the two fungal lines. On the contrary, at 4 and $37^{\circ} \mathrm{C}$, we detected only a low amount of MRE and no significant differences were observed between biomass of cured and WT lines. Curiously, the greatest measured biomass produced by the cured line of M. elongata NVP80 was observed at $4{ }^{\circ} \mathrm{C}$. Experimental results testing the effect of media type on fungal biomass production were also interesting. Indeed, we showed that the effect of MRE on their host seems to be influenced by the fungal diet. Indeed, diets with reduced sugars (1/10 ME and 1/10 PD) and/or higher amino acids (PE) appear to diminish the impact of MRE on the host biomass production.

In contrast to other Mollicutes lineages, $37^{\circ} \mathrm{C}$ does not seem to be an optimal temperature for the proliferation of 
Fig. 8 Transmission electron micrographs of MRE in the mycelium of $M$. humilis AD092. a-d Coccoid MRE cells (white arrowhead) are directly embedded in the fungal cytoplasm, where bacteria undergoing cell division were frequently observed (black arrowhead). MRE are in close proximity of lipid droplets (ld) that are abundant in hyphae of M. humilis. c, d Similar to $C a$. Moeniiplasma

glomeromycotorum, a thin homogeneous electron-dense layer is visible outside the bacterial cell membrane. Mitochondrion (m); nucleus (n); Scale bars, a $2 \mu \mathrm{m} ; \mathbf{b} 0.59 \mu \mathrm{m}$; c $0.26 \mu \mathrm{m} ; \mathbf{d} 0.25 \mu \mathrm{m}$

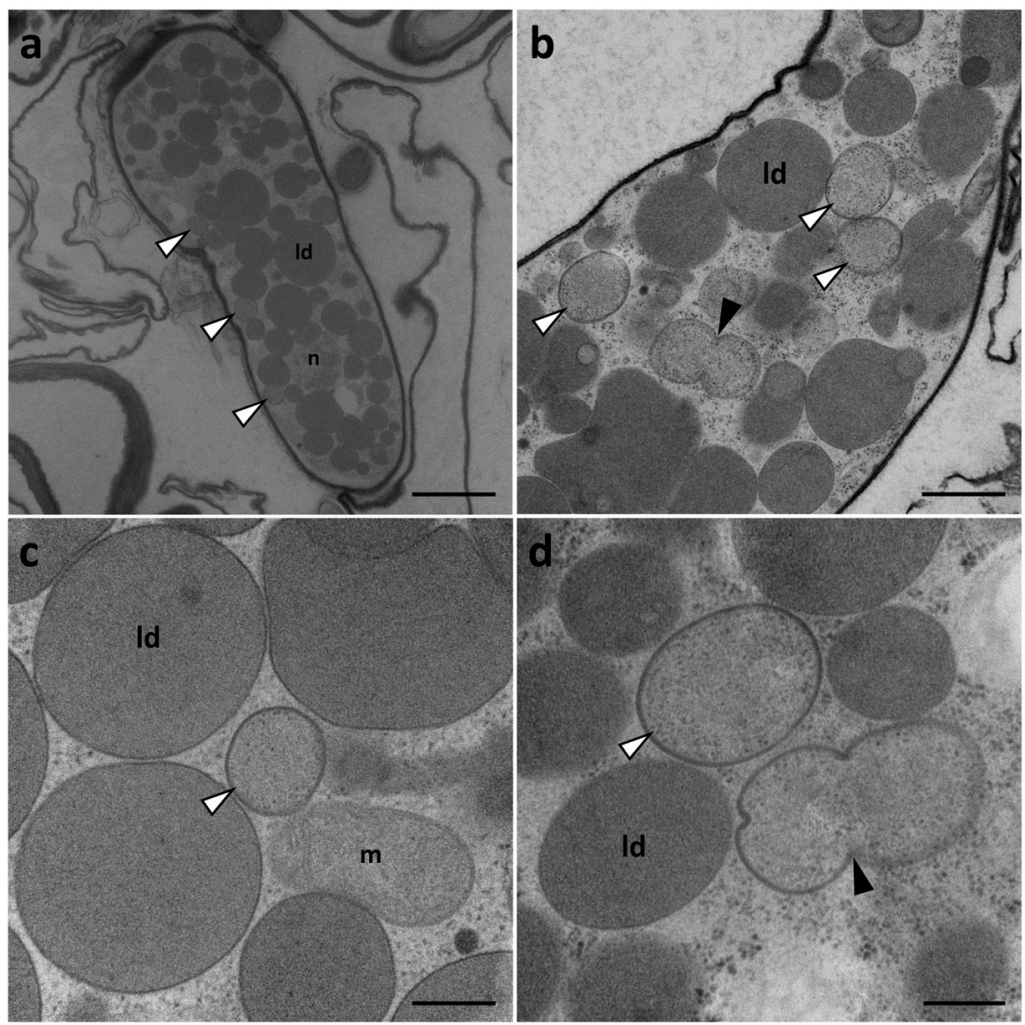

MRE. For example, many Mycoplasma species, closely related to MRE, show their best growth at $37^{\circ} \mathrm{C}$, likely reflecting their adaptation to "warm-blooded" vertebrate hosts [66]. Accordingly, MRE, as endosymbionts of soil fungi, might prefer lower temperatures such as $22^{\circ} \mathrm{C}$. Nevertheless, we cannot exclude a more indirect role of the temperature on MRE abundance and consider the possibility that at $37^{\circ} \mathrm{C}$ the fungal metabolism changes the MRE habitat. For example, it has been shown that temperature has effects on the metabolites and lipid content of Mortierella [67-69], which MRE are dependent upon. Thus, we hypothesize that metabolic changes by the host can modify the mycelial environment, creating conditions that are poorly suited for the growth and proliferation of MRE.

Toomer and colleagues [22] hypothesized that the pattern of intrahost diversity of MRE, more specifically $\mathrm{CaMg}$, and the process responsible for generating it, may mirror a parasitic lifestyle rather than a mutualistic one. Assuming that MRE are parasites and obligately dependent upon their fungal hosts for survival, they must impose some fitness costs to the fungus [22]. In this study, we provided the first empirical evidence of fitness costs experienced by the fungal host (Mortierellomycotina) in accommodating MRE. We interpret our results as an additional indication that MRE may be antagonistic to their fungal hosts. These endobacteria appear to be adapted to a non-lethal parasitic lifestyle in the mycelium of Mucoromycota. The hypothesis of MRE as parasites of fungi is consistent with their phylogenetic placement within Mollicutes, a group of bacteria that also includes parasites of arthropods, human, plants and vertebrates [57]. Nevertheless, it should also be noted that there are mutualistic symbionts within Mollicutes. For example, Spiroplasma (Mollicutes) confers protection to its Drosophila or aphid hosts against fungal pathogens [70], nematodes [71] and parasitoid wasps [72]. Xie and colleagues [73] also demonstrated that the male-killing Spiroplasma protects its Drosophila melanogaster host from parasitoid wasps. Interestingly, male-killer bacteria, which are maternally inherited bacteria that selectively induce death of male offspring of their host, are known to have a negative impact on the population they infect [74]. Thus, it appears that the presence of Spiroplasma may confer both benefits and detriments to its insect host. In line with that, we need to consider that even when an interaction appears to be more parasitic or negative for the fungal host, such as was shown for Mycoavidus cysteinexigens and Mortierella elongata [16], or MRE and Mortierellomycotina here, there may be context-dependent adaptive advantages at minimal cost to the fungus that have not yet been discovered [10]. For example, it may be possible that the MRE activity within Mortierellomycotina, as the one of M. cysteinexigens within M. elongata [16], may alter the volatile organic compound (VOC) profile of the host. These changes might confer protection against soil fungivorous invertebrates, 
such as from collembola or nematodes. Indeed, it is known that different fungal VOCs lead to different effects on soil fauna [75]. In line with that, we might hypothesize that the activity of MRE may modify the VOC profile, inhibiting (or promoting) the production of compounds that lure (or repel/ kill) fungal predators. Future studies on MRE and Mortierellomycotina will be able to unravel this microbial enigma.

\section{MRE and Mucoromycota: partners with an elusive past}

Mucoromycota are the best candidates for investigating interactions between fungi and endobacteria. Indeed, most examples of fungal endobacteria hitherto published are focused on fungi within Mucoromycota [11]. For example, the presence of BRE was reported in at least one representative of each of the three Mucoromycota subphyla [12, 14, 17]. Similarly, MRE were identified in Glomeromycotina [21] and afterwards in Endogonaceae [32], while the present study reports their presence in the mycelium of different species of Mortierellomycotina. The reasons why endobacteria are common dwellers of Mucoromycota fungi are still unknown. It has been hypothesized that a coenocytic mycelium, with only adventitious septa, a feature shared by members of Mucoromycota, might facilitate endobacterial colonization, movement and proliferation within the fungal host [9]. Interestingly, Mucoromycota share a number of other features, as follows: (1) they have an apical vesicle crescent hyphal tip structure [76]; (2) they have the ability to reproduce asexually and to produce macro-sporocarps; (3) they use plants as major host/substrate; and (4) they contain a high content of lipids in their mycelium [1]. One or more of these characters could contribute, together with the coenocytic mycelium, to make Mucoromycota the ideal niche for fungal endobacteria.

The interaction with endobacteria, and specifically with MRE, represents an additional ecological trait shared by the early-diverging group of Mucoromycota fungi. This intriguing association raises the question on the origin of this bacterial-fungal association. Recently, Bonfante and Desirò [11] provided two plausible scenarios to explain the origin of the interaction with MRE: an early and a late bacterial invasion. Previous divergence time estimations based on 16S rDNA phylogenetic reconstructions placed the origin of Entomoplasmatales and Mycoplasmatales at 410 MYA [34], whereas Mucoromycota are believed to have split from Dykaria some 550-700 MYA[16, 33]. Accordingly, MRE might have originated after their fungal hosts, in support of a late bacterial invasion hypothesis [11]. However, divergence dating of bacteria, particularly obligate endosymbionts, must be treated with caution, especially when data are missing. In this study, we showed that MRE in Glomeromycotina, Mortierellomycotina and Mucoromycotina are distinct from each other and form a monophyletic lineage. Moreover, they are more similar to each other than to other Entomoplasmatales and Mycoplasmatales taxa. Thus, these data are in line with the hypothesis advanced by Desirò and colleagues [32] and provide further support to an early bacterial invasion, whereby ancestral MRE colonized the mycelium of the common ancestor of Mucoromycota and were maintained, as a symplesiomorphic trait, in some lineages of Glomeromycotina, Mortierellomycotina and Mucoromycotina.

Regardless of when the MRE invasion of fungi happened, it is worth noting that MRE invaded and/or have been maintained only in specific fungal lineages. Indeed, MRE appear to be spread in Glomeromycotina, with multiple taxa hosting this group of endosymbionts [9, 21, 22]. Similarly, MRE are associated with different Mortierellomycotina lineages representing distinct phylogenetic groups, even though the frequency in this fungal subphylum appears to be dramatically lower than in Glomeromycotina [21, 22]. In contrast, published studies on MRE in Mucoromycotina are circumscribed to the genera Endogone and Jimgerdemannia (Endogonaceae), the only lineages in this subphylum that has been thoroughly investigated for the presence of MRE so far [32]. Our findings raise the question of whether MRE have preferences for particular Mucoromycota hosts, and whether MRE are present in other fungal lineages. We predict that a broader investigation of Mucoromycotina will reveal the presence of novel MRE in other fungal lineages.

Recently, Naito and colleagues [27] proposed a hostswitching event from animals to fungi to explain the origin of MRE as endosymbionts of fungi. Starting from this assumption, Bonfante and Desirò [11] hypothesized that soil invertebrates, such as arthropods, may associate with Mollicutes [66] and might have acted as vectors for the transmission of MRE during their grazing on fungal hyphae. The finding of MRE in Mortierellomycotina might further contribute in supporting the host-switching hypothesis, offering, however, a different interpretation. It is known that Mortierella has genes for the chitin metabolism and can utilize $\mathrm{N}$-acetylglucosamine [16, 77]. Moreover, Mortierella can degrade chitin [78], proliferate in presence of it [79] and can colonize, as described in this study, whole fragments of shrimp exoskeleton. Accordingly, we can hypothesize that MRE, which initially dwelled inside soil arthropods, might have been unwittingly "recruited" by a Mortierella ancestor growing inside invertebrate exoskeletons. Interestingly, zygomyceteous fungi of the phylum Zoopagomycota associate with arthropods as parasites or pathogens $[1,2,80]$ and could have facilitated in "recruiting" endobacteria from their 
hosts. For example, the entomopathogen fungus Pandora neoaphidis (Entomophthoromycotina) are known to attack pea aphids, which in turn can host Spiroplasma endobacteria [81]. Thus, it remains to be explored whether members of Zoopagomycota or other early-diverging fungal lineages harbor MRE.

\section{Conclusion}

This large-scale study on endobacteria of fungi revealed the presence of MRE in Mortierellomycotina, demonstrating that these endosymbionts are present within fungi across Mucoromycota and they may have lived in their common ancestor. Novel MRE 16S rDNA phylotypes were identified in Mortierellomycotina, contributing to increase the diversity of this already incredibly disparate group of intracellular bacteria of fungi and suggesting that additional MRE diversity is waiting to be discovered. By curing the fungus from its endobacteria and assessing fungal biomass production in different conditions, we showed that the fungal host experiences some fitness costs in accommodating its bacterial endosymbionts, which are affected by temperature and media type. These results provide the first experimental evidence of the role of MRE, suggesting their potential antagonistic role and adaptation to a non-lethal parasitic lifestyle in the mycelium of Mucoromycota. Finally, we conclude that fungi in Mortierellomycotina represent attractive model organisms for future studies aimed at exploring interactions between MRE and fungi. Indeed, the possibility of removing MRE from Mortierellomycotina, together with the easy-to-handle feature that distinguishes these fungi from the other MRE-associated Mucoromycota, make them excellent candidates for comparative metabolomics, proteomics, and transcriptomics functional studies. With the first tile of the MRE puzzle in place, now the rest of the tiles are waiting to be placed.

Acknowledgements We thank M Chilvers, G Doehring, A Gryganskyi, A Miller, KO'Donnell, A Porras-Alfaro, N Vande Pol, AM Vázquez Catoni, TJ Torres-Cruz, X Wang, CBS-Knaw Fungal Biodiversity Centre and NRRL Agricultural Research Service Culture Collection for fungal strains, and A Fryday, G Nderitu Mwangi and D Ojwang for soil samples. AD and GB were supported through the US National Science Foundation (NSF) DEB 1737898 and Michigan State University AgBioResearch NIFA project MICL02416. RR was supported through NSF grant DEB-1441728. AD and GB are grateful to the 'ZyGoLife consortium' for collaborations and training opportunities.

\section{Compliance with Ethical Standards}

Conflict of interest The authors declare that they have no conflict of interest.

\section{References}

1. Spatafora JW, Chang Y, Benny GL, Lazarus K, Smith ME, Berbee ML, et al. A phylum-level phylogenetic classification of zygomycete fungi based on genomescale data. Mycologia. 2016;108:1028-46.

2. Benny GL, Smith ME, Kirk PM, Tretter ED, White MM. Challenges and future perspectives in the systematics of Kickxellomycotina, Mortierellomycotina, Mucoromycotina, and Zoopagomycotina. In: Li D-W, editor. Biology of microfungi. Switzerland: Springer International Publishing; 2016. p. 65-126.

3. Nagy LG, Petkovits T, Kovács GM, Voigt K, Vágvölgyi C, Papp T. Where is the unseen fungal diversity hidden? A study of Mortierella reveals a large contribution of reference collections to the identification of fungal environmental sequences. New Phytol. 2011;191:789-94.

4. Wagner L, Stielow B, Hoffmann K, Petkovits T, Papp T, Vágvölgyi $\mathrm{C}$, et al. A comprehensive molecular phylogeny of the Mortierellales (Mortierellomycotina) based on nuclear ribosomal DNA. Persoonia. 2013;30:77-93.

5. Tedersoo L, Bahram M, Põlme S, Kõljalg U, Yorou NS, Wijesundera $\mathrm{R}$, et al. Global diversity and geography of soil fungi. Science. 2014;346:1256688.

6. Bonito G, Reynolds H, Robeson MS, Nelson J, Hodkinson BP, Tuskan G, et al. Plant host and soil origin influence fungal and bacterial assemblages in the roots of woody plants. Mol Ecol. 2014;23:3356-70.

7. Shakya M, Gottel N, Castro H, Yang ZK, Gunter L, Labbé J, et al. A multifactor analysis of fungal and bacterial community structure in the root microbiome of mature Populus deltoides trees. PLoS ONE. 2013;8:e76382.

8. Summerbell RC. Root endophyte and mycorrhizosphere fungi of black spruce, Picea mariana, in a boreal forest habitat: influence of site factors on fungal distributions. Stud Mycol. 2005;53:121-45.

9. Desirò A, Salvioli A, Ngonkeu EL, Mondo SJ, Epis S, Faccio A, et al. Detection of a novel intracellular microbiome hosted in arbuscular mycorrhizal fungi. ISME J. 2014;8:257-70.

10. Partida-Martínez LP. The fungal holobiont: evidence from early diverging fungi. Environ Microbiol. 2017;19:2919-23.

11. Bonfante P, Desirò A. Who lives in a fungus? The diversity, origins and functions of fungal endobacteria living in Mucoromycota. ISME J. 2017;11:1727-35.

12. Bianciotto V, Lumini E, Bonfante P, Vandamme P. 'Candidatus Glomeribacter gigasporarum' gen. nov., sp. nov., an endosymbiont of arbuscular mycorrhizal fungi. Int Syst Evol Microbiol. 2003;53:121-4.

13. Salvioli A, Ghignone S, Novero M, Navazio L, Venice F, Bagnaresi $\mathrm{P}$, et al. Symbiosis with an endobacterium increases the fitness of a mycorrhizal fungus, raising its bioenergetic potential. ISME J. 2016;10:130-44.

14. Ohshima S, Sato Y, Fujimura R, Takashima Y, Hamada M, Nishizawa T, et al. Mycoavidus cysteinexigens gen. nov., sp. nov., an endohyphal bacterium isolated from a soil isolate of the fungus Mortierella elongata. Int J Syst Evol Microbiol. 2016;66:2052-7.

15. Li Z, Yao Q, Dearth SP, Entler MR, Castro Gonzalez HF, Uehling $\mathrm{JK}$, et al. Integrated proteomics and metabolomics suggests symbiotic metabolism and multimodal regulation in a fungalendobacterial system. Environ Microbiol. 2017;19:1041-53.

16. Uehling J, Gryganskyi A, Hameed K, Tschaplinski T, Misztal PK, $\mathrm{Wu} \mathrm{S}$, et al. Comparative genomics of Mortierella elongata and its bacterial endosymbiont Mycoavidus cysteinexigens. Environ Microbiol. 2017;19:2964-83. 
17. Partida-Martinez LP, Monajembashi S, Greulich KO, Hertweck C. Endosymbiont-dependent host reproduction maintains bacterialfungal mutualism. Curr Biol. 2007;17:773-7.

18. Partida-Martínez LP, Hertweck C. A gene cluster encoding rhizoxin biosynthesis in Burkholderia rhizoxinica, the bacterial endosymbiont of the fungus Rhizopus microsporus. Chembiochem. 2007;8:41-45.

19. MacDonald RM, Chandler M, Mosse B. The occurrence of bacterium-like organelles in vesiculararbuscular mycorrhizal fungi. New Phytol. 1982;90:659-63.

20. Bonfante P, Scannerini S. Cytological observations on the mycorrhiza Endogone flammicorona-Pinus strobus. Allionia. 1977;22:23-34

21. Naumann M, Schüßler A, Bonfante P. The obligate endobacteria of arbuscular mycorrhizal fungi are ancient heritable components related to the Mollicutes. ISME J. 2010;4:862-71.

22. Toomer KH, Chen X, Naito M, Mondo SJ, den Bakker HC, VanKuren NW, et al. Molecular evolution patterns reveal life history features of mycoplasma-related endobacteria associated with arbuscular mycorrhizal fungi. Mol Ecol. $2015 ; 24: 3485-3500$.

23. Ligrone R. Ultrastructure of a fungal endophyte in Phaeoceros laevis (L.) Prosk. (Anthocerotophyta). Bot Gaz. 1988;149:92-100.

24. Schmid E, Oberwinkler F. Mycorrhiza-like interaction between the achlorophyllous gametophyte of Lycopodium clavatum $\mathrm{L}$. and its fungal endophyte studied by light and electron microscopy. New Phytol. 1993;124:69-81.

25. Desirò A, Naumann M, Epis S, Novero M, Bandi C, Genre A, et al. Mollicutes-related endobacteria thrive inside liverwortassociated arbuscular mycorrhizal fungi. Environ Microbiol. 2013;15:822-36.

26. Naito M, Desirò A, Gonzales JB, Tao G, Morton JB, Bonfante P, et al. Candidatus Moeniiplasma glomeromycotorum', an endobacterium of arbuscular mycorrhizal fungi. Int $\mathrm{J}$ Syst Evol Microbiol. 2017;67:1177-84.

27. Naito M, Morton JB, Pawlowska TE. Minimal genomes of mycoplasma-related endobacteria are plastic and contain hostderived genes for sustained life within Glomeromycota. Proc Natl Acad Sci USA. 2015;112:7791-6.

28. Torres-Cortés G, Ghignone S, Bonfante P, Schüßler A. Mosaic genome of endobacteria in arbuscular mycorrhizal fungi: transkingdom gene transfer in an ancient mycoplasma-fungus association. Proc Natl Acad Sci USA. 2015;112:7785-90.

29. Naito M, Pawlowska TE. The role of mobile genetic elements in evolutionary longevity of heritable endobacteria. Mob Genet Elem. 2016a;6:e1136375.

30. Naito M, Pawlowska TE. Defying Muller's ratchet: heritable endobacteria escape extinction through recombination and genome plasticity. mBio. 2016b;7:e02057-15.

31. Desirò A, Rimington WR, Jacob A, Vande Pol N, Smith ME, Trappe JM, Bidartondo MI, Bonito G. Multigene phylogeny of Endogonales, an early diverging lineage of fungi associated with plants. IMA Fungus. 2017;8:245-57.

32. Desirò A, Faccio A, Kaech A, Bidartondo MI, Bonfante P. Endogone, one of the oldest plant-associated fungi, host unique Mollicutes-related endobacteria. New Phytol. 2015;205:1464-72.

33. Chang Y, Wang S, Sekimoto S, Aerts AL, Choi C, Clum A, et al. Phylogenomic analyses indicate that early fungi evolved digesting cell walls of algal ancestors of land plants. Genome Biol Evol. 2015;7:1590-601.

34. Maniloff J. Phylogeny and evolution. In: Razin S, Herrmann R, editors. Molecular biology and pathogenicity of Mycoplasmas. New York, NY: Kluwer Academic/Plenum Publishers; 2002. p. 31-44.

35. Sato Y, Narisawa K, Tsuruta K, Umezu M, Nishizawa T, Tanaka $\mathrm{K}$, et al. Detection of betaproteobacteria inside the mycelium of the fungus Mortierella elongata. Microbes Environ. 2010;25:321-4.

36. Warcup JH. The soil-plate method for isolation of fungi from soil. Nature. 1950;166:117-8.

37. Shirouzu T, Hirose D, Tokumasu S. Biodiversity survey of soilinhabiting mucoralean and mortierellalean fungi by a baiting method. T Mycol Soc Jpn. 2012;53:33-39.

38. Bonito G, Hameed K, Ventura R, Krishnan J, Schadt CW, Vilgalys R. Isolating a functionally relevant guild of fungi from the root microbiome of Populus. Fungal Ecol. 2016;22:35-42.

39. Doyle J. DNA protocols for plants: a CTAB total DNA isolation. In: Hewitt GM, Johnston A, editors. Molecular techniques in taxonomy. New York, NY: Springer; 1991. p. 283-93.

40. Gardes M, Bruns TD. ITS primers with enhanced specificity for basidiomycetes - application to the identification of mycorrhizae and rusts. Mol Ecol. 1993;2:113-8.

41. Vilgalys R, Hester M. Rapid genetic identification and mapping of enzymatically amplified ribosomal DNA from several Cryptococcus species. J Bacteriol. 1990;172:4238-46.

42. Naumann M. Tracing endobacteria in arbuscular mycorrhizal fungi: identification and molecular characterization of a new bacterial taxon. Ph.D. Dissertation. University of Turin, Turin, Italy; 2009.

43. Turner S, Pryer KM, Miao VPW, Palmer JD. Investigating deep phylogenetic relationships among cyanobacteria and plastids by small subunit rRNA sequence analysis. J Eukaryot Microbiol. 1999;46:327-38

44. Kearse M, Moir R, Wilson A, Stones-Havas S, Cheung M, Sturrock S, et al. Geneious Basic: an integrated and extendable desktop software platform for the organization and analysis of sequence data. Bioinformatics. 2012;28:1647-9.

45. Katoh K, Standley DM. MAFFT multiple sequence alignment software version 7: improvements in performance and usability. Mol Biol Evol. 2013;30:772-80.

46. Edgar RC. MUSCLE: a multiple sequence alignment method with reduced time and space complexity. BMC Bioinforma. 2004;5:113.

47. Castresana J. Selection of conserved blocks from multiple alignments for their use in phylogenetic analysis. Mol Biol Evol. 2000; $17: 540-52$

48. Darriba D, Taboada GL, Doallo R, Posada D. jModelTest 2: more models, new heuristics and parallel computing. Nat Methods. 2012;9:772.

49. Ronquist F, Teslenko M, van der Mark P, Ayres DL, Darling A, Höhna S, et al. MrBayes 3.2: efficient Bayesian phylogenetic inference and model choice across a large model space. Syst Biol. 2012;61:539-42.

50. Stamatakis A. RAxML version 8: A tool for phylogenetic analysis and post-analysis of large phylogenies. Bioinformatics. 2014;30:1312-3.

51. Kosakovsky Pond SL, Posada D, Gravenor MB, Woelk CH, Frost SDW. Automated phylogenetic detection of recombination using a genetic algorithm. Mol Biol Evol. 2006;23:1891-901.

52. Caporaso JG, Lauber CL, Walters WA, Berg-Lyons D, Huntley J, Fierer N, et al. Ultra-high-throughput microbial community analysis on the Illumina HiSeq and MiSeq platforms. ISME J. 2012;6:1621-4.

53. Lundberg DS, Yourstone S, Mieczkowski P, Jones CD, Dangl JL. Practical innovations for high-throughput amplicon sequencing. Nat Methods. 2013;10:999-1002.

54. Desirò A, Salvioli A, Bonfante P. Investigating the endobacteria which thrive in arbuscular mycorrhizal fungi. In: Martin F, Uroz S, editors. Microbial Environmental Genomics (MEG). Methods in Molecular Biology. New York, NY, USA: Humana Press; 2016. p. 29-53. 
55. $\mathrm{R}$ Core Team. R: a language and environment for statistical computing. Vienna, Austria: R Foundation for Statistical Computing; 2017. P.

56. Smith ME, Gryganskyi A, Bonito G, Nouhra E, Moreno-Arroyo B, Benny G. Phylogenetic analysis of the genus Modicella reveals an independent evolutionary origin of sporocarp-forming fungi in the Mortierellales. Fungal Genet Biol. 2013;61:61-68.

57. Razin S, Yogev D, Naot Y. Molecular biology and pathogenicity of mycoplasmas. Microbiol Mol Biol R. 1998;62:1094-156.

58. Lo N, Paraskevopoulos C, Bourtzis K, O'Neill SL, Werren JH, Bordenstein SR, et al. Taxonomic status of the intracellular bacterium Wolbachia pipientis. Int $\mathrm{J}$ Syst Evol Microbiol. 2007;57:654-7.

59. Partida-Martinez LP, Hertweck C. Pathogenic fungus harbours endosymbiotic bacteria for toxin production. Nature. 2005;437:884-8.

60. Lastovetsky OA, Gaspar ML, Mondo SJ, LaButti KM, Sandor L, Grigoriev IV, et al. Lipid metabolic changes in an early divergent fungus govern the establishment of a mutualistic symbiosis with endobacteria. Proc Natl Acad Sci USA. 2016;113:15102-7.

61. Mondo SJ, Lastovetsky OA, Gaspar ML, Schwardt NH, Barber $\mathrm{CC}$, Riley R, et al. Bacterial endosymbionts influence host sexuality and reveal reproductive genes of early divergent fungi. Nat Commun. 2017;8:1843.

62. Salvioli A, Chiapello M, Fontaine J, Hadj-Sahraoui AL, Grandmougin-Ferjani A, Lanfranco L, et al. Endobacteria affect the metabolic profile of their host Gigaspora margarita, an arbuscular mycorrhizal fungus. Environ Microbiol. 2010;12:2083-95.

63. Vannini C, Carpentieri A, Salvioli A, Novero M, Marsoni M, Testa L, et al. An interdomain network: the endobacterium of a mycorrhizal fungus promotes antioxidative responses in both fungal and plant hosts. New Phytol. 2016;211:265-75.

64. Venice F, de Pinto MC, Novero M, Ghignone S, Salvioli A, Bonfante P. Gigaspora margarita with and without its endobacterium shows adaptive responses to oxidative stress. Mycorrhiza. 2017. https://doi.org/10.1007/s00572-017-0790-z

65. Lumini E, Bianciotto V, Jargeat P, Novero M, Salvioli A, Faccio A, et al. Presymbiotic growth and sporal morphology are affected in the arbuscular mycorrhizal fungus Gigaspora margarita cured of its endobacteria. Cell Microbiol. 2007;9:1716-29.

66. Tully JG, Bové JM, Laigret F, Whitcomb RF. Revised taxonomy of the class Mollicutes: proposed elevation of a monophyletic cluster of arthropod-associated Mollicutes to ordinal rank (Entomoplasmatales ord. nov.), with provision for familial rank to separate species with nonhelical morphology (Entomoplasmataceae fam. nov.) from helical species (Spiroplasmataceae), and emended descriptions of the order Mycoplasmatales, family Mycoplasmataceae. Int J Syst Evol Microbiol. 1993;43:378-85.

67. Dyal SD, Bouzidi L, Narine SS. Maximizing the production of $\gamma$ linolenic acid in Mortierella ramanniana var. ramanniana as a function of $\mathrm{pH}$, temperature and carbon source, nitrogen source, metal ions and oil supplementation. Food Res Int. 2005;38:815-29.

68. Lindberg AM, Molin G. Effect of temperature and glucose supply on the production of polyunsaturated fatty acids by the fungus Mortierella alpina CBS 343.66 in fermentor cultures. Appl Microbiol Biot. 1993;39:450-5.

69. Weinstein RN, Montiel PO, Johnstone K. Influence of growth temperature on lipid and soluble carbohydrate synthesis by fungi isolated from fellfield soil in the maritime Antarctic. Mycologia. 2000;92:222-9.

70. Łukasik P, van Asch M, Guo H, Ferrari J, Godfray CJ. Unrelated facultative endosymbionts protect aphids against a fungal pathogen. Ecol Lett. 2013a;16:214-8.

71. Cockburn SN, Haselkorn TS, Hamilton PT, Landzberg E, Jaenike J, Perlman SJ. Dynamics of the continent-wide spread of a Drosophila defensive symbiont. Ecol Lett. 2013;16:609-16.

72. Xie J, Vilchez I, Mateos M. Spiroplasma bacteria enhance survival of Drosophila hydei attacked by the parasitic wasp Leptopilina heterotoma. PLoS One. 2010;5:e12149.

73. Xie J, Butler S, Sanchez G, Mateos M. Male killing Spiroplasma protects Drosophila melanogaster against two parasitoid wasps. Heredity. 2014;112:399-408.

74. Engelstädter J, Hurst GD. The impact of male-killing bacteria on host evolutionary processes. Genetics. 2007;175:245-54.

75. Werner S, Polle A, Brinkmann N. Belowground communication: impacts of volatile organic compounds (VOCs) from soil fungi on other soil-inhabiting organisms. Appl Microbiol Biot. 2016;100:8651-65.

76. Fisher KE, Roberson RW. Hyphal tip cytoplasmic organization in four zygomycetous fungi. Mycologia. 2016;108:533-42.

77. Kim YJ, Zhao Y, Oh KT, Nguyen VN, Park RD. Enzymatic deacetylation of chitin by extracellular chitin deacetylase from a newly screened Mortierella sp. DY-52. J Microbiol Biotechn. 2008;18:759-66.

78. De Boer W, Gerards S, Gunnewiek PK, Modderman R. Response of the chitinolytic microbial community to chitin amendments of dune soils. Biol Fert Soils. 1999;29:170-7.

79. Debode J, De Tender C, Soltaninejad S, Van Malderghem C, Haegeman A, Van der Linden I, et al. Chitin mixed in potting soil alters lettuce growth, the survival of zoonotic bacteria on the leaves and associated rhizosphere microbiology. Front Microbiol. 2016;7:565.

80. Humber RA. Entomophthoromycota: a new overview of some of the oldest terrestrial fungi. In: $\mathrm{Li} \mathrm{D}-\mathrm{W}$, editor. Biology of microfungi. Switzerland: Springer International Publishing; 2016. p. $127-45$.

81. Łukasik P, Guo H, Asch M, Ferrari J, Godfray HC. Protection against a fungal pathogen conferred by the aphid facultative endosymbionts Rickettsia and Spiroplasma is expressed in multiple host genotypes and species and is not influenced by coinfection with another symbiont. J Evol Biol. 2013b;26:2654-61. 